\title{
ECG Paper Records Digitization through Image Processing Techniques
}

\author{
Deepak Kumar Garg* \\ Amity School of \\ Engineering \& Technology \\ Amity University Noida, \\ Uttar Pradesh India
}

\author{
Diksha Thakur \\ Amity School of \\ Engineering \& Technology \\ Amity University Noida, \\ Uttar Pradesh India
}

\author{
Seema Sharma \\ Assistant Professor \\ Amity School of \\ Engineering \& Technology \\ Amity University Noida, \\ Uttar Pradesh India
}

\author{
Shweta Bhardwaj \\ Assistant Professor \\ Amity School of \\ Engineering \& Technology \\ Amity University Noida, \\ Uttar Pradesh India
}

\begin{abstract}
Electrocardiogram (ECG) is one of the most practiced methods to detect any abnormalities in heart function. ECG results are available as paper records. This leads to Archival and retrieval of patient's paper ECG records as a common practice for diagnosis of ailments. This process requires large storage space and extensive manual effort. This paper discusses method that involves processing of ECG paper records by an efficient and iterative set of digital image processing techniques for the conversion of ECG paper image data to time series digitized signal form, resulting in convenient storage and retrieval of ECG information. The method involves calculation of Heart rate, QRS Width and Stability (variation in R-R peaks) from the extracted signal. Comparison of the above calculated parameters with the manually calculated parameters shows an accuracy of $96.4 \%$, hence proves the effectiveness of the process. This paper also proposes the development of fuzzy based ECG diagnosis system that assists the doctor in diagnosis.
\end{abstract}

\section{General Terms}

Biomedical Engineering, Electrocardiogram (ECG), Image Processing

\section{Keywords}

ECG, Scanned Images, Heart Rate, QRS Width, Stability, Digitization, Fuzzy, Biomedical Signal Processing

\section{INTRODUCTION}

The electrocardiogram (ECG) is a graphical representation of the electrical activity of the heart and is obtained by connecting specially designed electrodes to the surface of the body. Normally, ECG has to be printed on a thermal paper for further physical inspection by medical practitioner. This leads to large volume of ECG reports that results in a tedious and error prone process of examining and retrieval of the same. Further these bulk ECG records are very hard to be shared among doctors. Digitizing the ECG paper signal will help to solve storage problem. It is also cost effective when comes to sharing and diagnosis.

It is to be noted that printed ECG reports includes some characters as well as annotations, while taking an image of such ECG strips it is desirable to remove these printed characters. Efficient noise removal and image enhancing algorithms are also required so as to increase overall digitization process accuracy.

Previous research work in the direction of digitizing the ECG paper records is referred in [2-8], in which morphological approach [2] [6] is used to remove the background grid from the ECG. An XOR operation is performed on the binary image's first periodic distance vertical direction (PDVD) and its shifted version. For it to work successfully, correct estimation of PDVD is necessary which strongly depends on the binarization of the image. Two approaches [3] are used namely spatial oriented approach and frequency oriented approach. Software [4] [5] is also designed for this purpose. It needs some manual intervention in selecting ECG signal and ECG signal printed on pure black and white background grid can result in incorrect digitization.

This paper proposes an effective algorithm for extracting the digitized signal from ECG paper strips. Hough transform [1] is used for de-skewing the scanned/captured images. With the combination of other image processing techniques, this algorithm presents an iterative process for the desired purpose. Further, a Fuzzy Logic Based ECG diagnosis system is proposed. To validate this technique it is compared against the original data (manually read from ECG record). The digitized signal can also be used in computer adaptive ECG diagnosis systems.

The organization of the paper is as follows.

Section I provides introduction to the research topic. Section II presents methodology and algorithm for converting ECG images (Scanned/captured) to Digital Signal. Section III, discusses details of the Method. In Section IV results are discussed. In Section V a Fuzzy Logic based ECG diagnostic System is proposed and Finally, Section VI presents conclusion and future work of this research.

\section{METHOD}

ECG strips (printed on a thermal paper) are scanned and scanned images are enhanced by applying de-skewing operation using Hough transformation and noise removal is done by using median filtration. The skewness and noise are some normal characteristics that may come in any scanned image and need to be avoided in order to acquire better results. The grid is removed from the scanned ECG images by using color segmentation. Color based segmentation can result in isolated pixels that represent printed characters or noise so further region based segmentation is applied. Final processed ECG signal is obtained by interpolating the pixel values and the signal is represented by a specific signal pixel per column. On the final processed signal Compression technique is applied to reduce the size of the ECG signal file for efficient storage and transmission.

The overall algorithmic steps are shown in Figure 1 below 


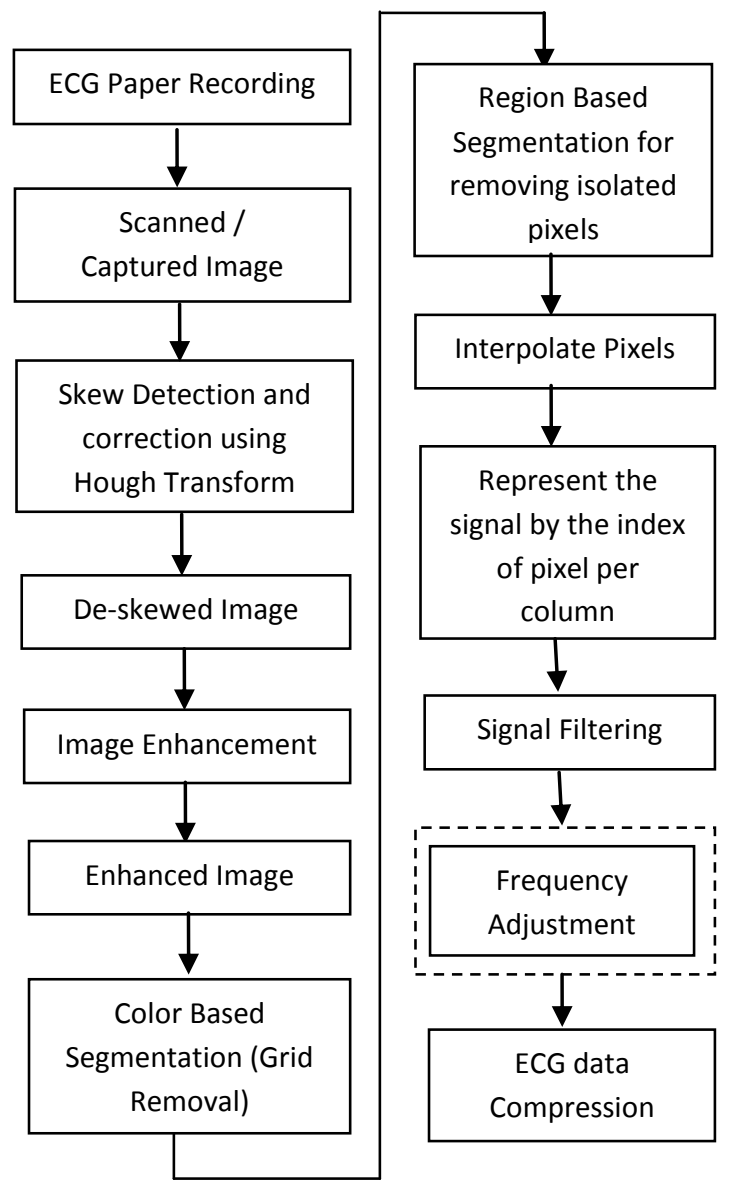

Fig 1: ECG Paper Record Digitization Process

\section{DETAILS}

The detailed explanation of various steps in the algorithm is as follows:-

\subsection{Scanning}

ECG paper recordings need to be scanned. Scanning resolution can be 600/300/200 dpi (dots per inch). Preferred algorithm for the image compression is JPEG. The image can also be obtained from a digital camera used in mobile phones.

Figure 2 represents a scanned ECG paper recording.

\subsection{De-Skewing}

Skew is a common phenomena that can appear in a scanned image. Skewing rotates the image to an angle, resulting in a rotated image. Hough Transform has been used to de-skew image. Skew angle is calculated using the background grid lines of scanned ECG image.

\subsection{Image Enhancement}

This step enhances the ECG image by making the signal lines sharper. Laplacian filtering is applied for making background noise lighter then the main ECG signal. This is followed by setting a threshold. Threshold value is chosen by comparing between noise pixels and pixels representing actual ECG signal. If ECG signal pixels values close to the threshold then pixels will be made darker by subtracting a fix value. Whereas the noise pixels values, close to the threshold, will be made lighter by adding a fix value.
Therefore, the resulting image will contain distinct ECG signal in the image.

\subsection{Color Based Segmentation}

This step is applied to remove the background grid of an ECG. Background grid is usually of lighter shade of color than the actual signal waveform. In this step ECG image is processed column by column. The darkest pixels are extracted in each column and are replaced by pure black pixel and rest of the pixels as pure white. It produces a binarized image however this may lead to extraction of undesired printed character as well.

Figure 2 (b) shows the output of color based segmentation; it has some printed characters also.

\subsection{Region Based Segmentation}

This step removes the isolated pixels that do not represent the signal (printed characters). This step comprises of the following steps:-

Step 0: Remove the frame of the image that is border of the input image.

Step 1: Scan the input ECG image column by column.

Step 2: Repeat for each column:

a. Extract the black pixels (pixels that represent the signal).

b. In case of the presence of isolated pixels (not likely to happen with the color based segmentation), delete them.

c. If there is more than one black pixel:

i. Algorithm first checks the previous and the next columns, if they contain only one pixel, then for the current column select the pixel that likely goes with the flow of the signal pattern.

ii. If the next column contains the same problem of having more than one black pixel, then for the current column select the middle pixel that lies exactly in the middle of the black pixel pattern.

Step 3: Save the index of the picked pixel.

The process of choosing the middle value in step 2 is performed by the following algorithm:

Step 0: get the centers of different regions

Step 1: separate regions and compute the centroid of each region

\section{1a: Set threshold value of region separation}

If current_point > previous_ample + threshold value

(if true, then current region is separated)

add center(average of first and last point in the region)

else (we need to check whether it is a last region)

add center( average of first and last point in the region)

Step 4: Pick centre point value which is the closest to the previous column pixel index. For this centres calculated in step 2 are used. We find the center that has the minimum difference with the previous column pixel index and that is our next center.

Figure 2 (c) shows the output of this step, the red line is actual detected signal. It is to be noted here that the printed 
characters (patient id....) are still there but do not have any significance over the actual detected signal (red line).

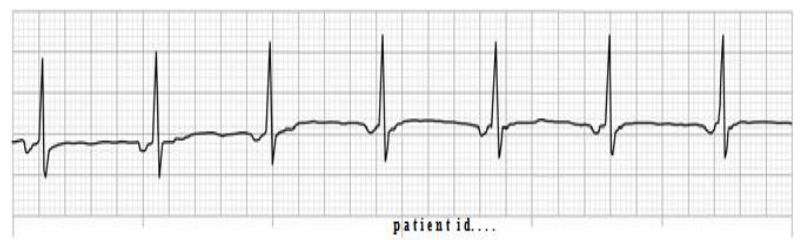

(a)

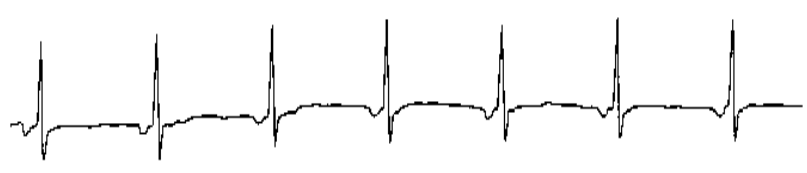

patientid....

(b)

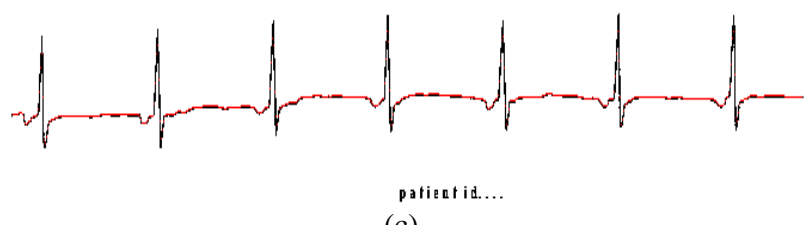

(c)

Fig 2: steps in algorithm

\subsection{Signal Representation}

Since each column is governed by a single pixel so the signal is represented by the corresponding column pixel index.

Finally the signal is saved as a txt file

\subsection{Median Filtration}

Actual ECG signal recorded by an ECG machine can be degraded due to presence of noise. Various sources of noise can be [10]:

- Item Power Line Interface $(50 \mathrm{~Hz}$ or $60 \mathrm{~Hz}$ noise from power lines)

- Baseline wander (low frequency noise)

- Muscle noise

- Other interference (i.e., radio frequency noise from other equipment)

Noisy signal can result into misdiagnosis so it is desirable to remove noise from the actual ECG signal as much as possible. A noisy ECG signal image is taken and the performance of various filters including median filter, FIR or IIR type of filters are compared and it is found that median filter has best result both by visual inspection and by quantitative measurement (by Signal to Noise Ratio). Following table shows the SNR comparison:

Table 1. Comparison of different filters in terms of SNR(dB)

\begin{tabular}{ccc}
\hline \hline Filters & $\begin{array}{c}\text { Noise Level } \\
\text { (noise with 10\%) }\end{array}$ & $\begin{array}{c}\text { Noise Level } \\
\text { (noise with 20\%) }\end{array}$ \\
\hline \hline w/o filter & 24.3981 & 16.3517 \\
Remez & 51.8763 & 51.4654 \\
Yulewalk & 58.7657 & 58.4501 \\
Median & 66.8568 & 65.7764
\end{tabular}

\subsection{Frequency Adjustment}

This step is necessary to perform in order to implement automatic computer adaptive ECG diagnosis system (as proposed in section $\mathrm{V}$ of this research paper). The aim is to adjust ECG signal frequency so that various feature extraction algorithms (such as QRS width detection, stability measurement etc.) can work efficiently. This step is also necessary in cases where the final extracted signal is of very low amplitude due to extreme noise.

The signal amplitude is enhanced by a magnification factor: Magnificaiton_factor $=a m p \_r e q u i r e d /$ original_amp (1) Where, amp stands for the amplitude of the signal.

\subsection{ECG Data Compression}

The aim is to achieve higher compression rate while not compromising in signal reconstruction. ECG data compression technique can be measured in terms of ratio of compressed data size to the original data size, also known as compression ratio (CR); execution time, the processing time required for compression and de-compression of ECG data; measuring the percent mean-square difference (PRD).

$$
P R D=100 \times \sqrt{\left(\sum_{i=1}^{n}(O(i)-R(i))^{2}\right) \div \sum_{i=1}^{n}(O(i))^{2}}
$$

Where $O$ is the original signal and $R$ is the reconstructed signal.

The final digitized ECG signal obtained from frequency adjustment step can be represented as set of integers and further can be stored in txt files. These txt files containing ECG signal are compressed using ZIP algorithm. ZIP compression offers smaller processing time and moderate CR thus reducing the storage requirement and faster data sharing over the network.

\section{RESULTS AND DISCUSSIONS}

For the analysis, ECG database consisting of 12 leads ECG paper records of 20 patients. Traces of these scanned ECG leads consisting $30 \mathrm{big}$ squares were created. Each square is of 0.20 seconds and $0.5 \mathrm{mVolts}$.

In [2] digitization technique significantly depends on scanning resolution. Poor scanning of ECG paper records may lead to loss of vertical grids that can result to erroneous PDVD calculation. In our method, background grids are removed using color based segmentation technique. Our Method shows a higher accuracy $(96.4 \%)$ in digitization process than the process used in [6]. Our method also has a Frequency Adjustment step for efficient computer adaptive ECG diagnosis and further the integration of ECG data compression with digitization leads to lesser storage requirement.

To evaluate and calculate the accuracy of this digitization process, Heart Rate, QRS Width and Stability (variation in R$\mathrm{R}$ peaks) have been used. For the calculation of above parameters self written programs are used, in which QRS Width detection program is based on a Pascal program [9]. 
Table 2. Comparison Based On Various ECG Features

\begin{tabular}{ccccccc}
\hline $\begin{array}{c}\text { Scanne } \\
\text { d ECG } \\
\text { Image }\end{array}$ & $\begin{array}{c}\text { Heart } \\
\text { Rate } \\
\text { Detected }\end{array}$ & $\begin{array}{c}\text { Heart } \\
\text { Rate } \\
\text { Observe } \\
\text { d on } \\
\text { printed } \\
\text { ECG }\end{array}$ & $\begin{array}{c}\text { QRS } \\
\text { Widt } \\
\text { h } \\
\text { Detec } \\
\text { ted }\end{array}$ & $\begin{array}{c}\text { QRS } \\
\text { Width } \\
\text { Observe } \\
\text { d on } \\
\text { printed } \\
\text { ECG }\end{array}$ & $\begin{array}{c}\text { Stability } \\
\text { Detected }\end{array}$ & $\begin{array}{c}\text { Stability } \\
\text { Observe } \\
\text { d on } \\
\text { printed } \\
\text { ECG }\end{array}$ \\
\hline 1 & 73 & 78 & 87 & 88 & 28 & 28 \\
2 & 54 & 56 & 118 & 120 & 130 & 132 \\
3 & 56 & 59 & 128 & 125 & 132 & 132 \\
4 & 60 & 63 & 104 & 104 & 12 & 11 \\
5 & 98 & 102 & 104 & 102 & 24 & 24 \\
6 & 117 & 122 & 88 & 88 & 150 & 144 \\
$7 *$ & $100 *$ & 70 & $104 *$ & 80 & $60 *$ & 24 \\
& & & & & $*$ corrupted signal
\end{tabular}

The algorithm is able to achieve about $96.4 \%$ accuracy in converting an ECG paper record to a digital signal.

\section{PROPOSED FUZZY LOGIC BASED DIAGNOSTIC SYSTEM}

The digitized output of the above method can be used for ECG diagnosis. The importance of fuzzy based system is that it is inherently robust since it does not require precise, noisefree inputs and can be programmed to fail safely if a feedback sensor quits or is destroyed. The output control is a smooth control function despite a wide range of input variations that is a noisy ECG signal can be diagnosed accurately.

This paper proposes a Fuzzy Logic Based Diagnosis System that has rule set for heart diseases, Facts as the features of ECG signal i.e. Heart Rate, QRS Width and Stability and sets of these Facts. The input ECG image is digitized by the above algorithm and its output (a compressed signal file containing sets of indexes of column pixels) is fed to the fuzzy diagnosis system. This leads to first decompression followed by features extraction, these features are used to calculate membership of the input signal and finally it presents the diagnosis of the signal.

Figure 3 shows a proposed flow chart of the system:-

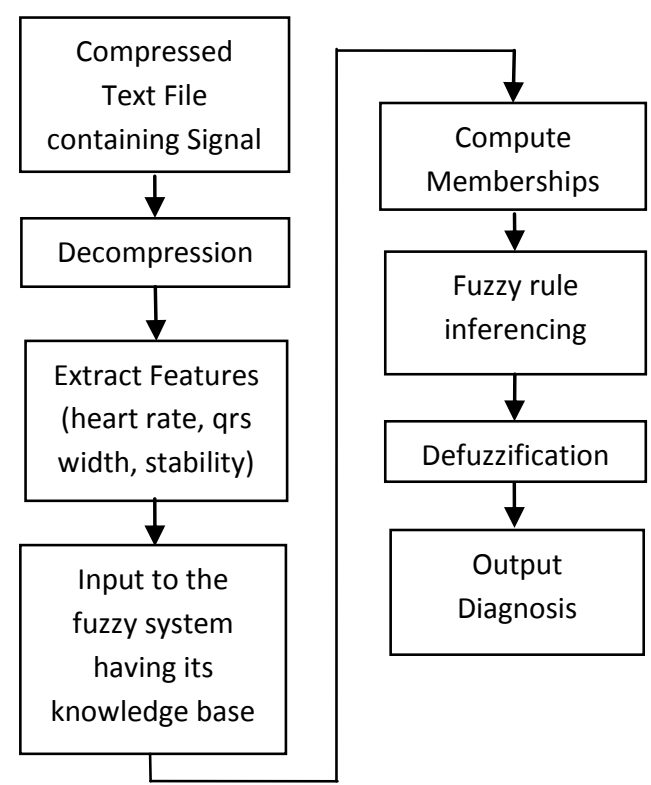

Fig 3: Proposed Fuzzy Expert System

\section{CONCLUSION AND FUTURE WORK}

An efficient method for extraction and digitization of ECG signal from various sources such as thermal ECG printouts, scanned ECG and captured ECG images from devices is proposed. The methodology produced a reasonably accurate waveform that is free from printed character and as tested through heart rate, qrs width and stability calculations.

Further work is in progress to enhance the overall efficiency of the method and also developing a fuzzy based expert system that can assist a doctor in diagnosis and generating automatic diagnosis reports as well.

\section{REFERENCES}

[1] Rafael C. Gonzalez and Richard E. Woods, Digital Image Processing, 3rd Edition, Prentice Hall, 2008.

[2] Tsair Kao, Len-Jon hwang, Yui Han Lin, Tzong huei Lin, Chia Hung Hsio, Computer Analysis of Electrocardiograms from ECG paper Recordings Proceeding of 23rd annual EMBS international conferences, Istanbul, Turkey, 2001.

[3] TW Shen, TF LaioImage, Processing on ECG Chart for ECG Signal Recovery, Tzu Chi University, Hualien, Taiwan Computers in Cardiology 2009; 36:725728

[4] Ricardo Baeza, Electrocardiogram digitization: a practical perspective on the usefulness of a new tool to convert paper electrocardiograms into digital waveform, Journal of Electrocardiology, vol. 38, issue 4, pp. 321323, October 2005

[5] Fabio Badilini, Tanju Erden, Wojciech Zareba, and Arthur J. Moss, ECGscan: a method for conversion of paper electrocardiographic printouts to digital electrocardiographic files, Journal of Electrocardiology, vol. 38, issue 4, pp. 310-318, October 2005.

[6] Prashanth Swamy, Srinivasan Jayaraman, and M.Girish Chandra, An Improved Method for Digital Time Series Signal Generation from Scanned ECG Records, International Conference on Bioinformatics and Biomedical Technology, pp. 400-403, 2010.

[7] H.K. Bhullar, J.C. Fothergill and D.P.de Bono, Computer based techniques for the optimal extraction of medical data from graphical paper records, Medical Imaging: Image Processing and Analysis, pp. 411-412, Mar 1992.[IEEE Colloquium]

[8] A. R. Gomes e Silva, H.M. de Oliveira, R.D. Lins., Converting ECG and other paper legated biomedical maps into digital signals, XXV Simpsiov Brasileiro de Telecomunicaes, Setembro 3-6, Recife PE, Brasil.

[9] Pascal program written by W.A.H. Engelse and C. Zeelenberg, A single scan algorithm for QRS detection and feature extraction, www.physionet.org/physiotools/wag/sqrs-1.htm

[10] Techniques-for-accurate-ECG-signal-processing, ECG Filtering, http://www.eetimes.com/design/medicaldesign/4213140/Techniques-foraccurate-ECG-signal processing 